\title{
TENSÃO INTERFACIAL DINÂMICA DE SOLUÇÕES CONTENDO ASFALTENOS DO PETRÓLEO E ÁLCOOIS
}

\author{
L. S. MARTINS ${ }^{1}$, R. G. SANTOS ${ }^{1}$ \\ ${ }^{1}$ Centro Universitário da FEI, Departamento de Engenharia Química \\ E-mail para contato: rgsantos@fei.edu.br, lilian_smartins@yahoo.com.br
}

\begin{abstract}
RESUMO - Asfaltenos compreendem os compostos mais polares e interfacialmente ativos do petróleo e tem sido apontado como o principal responsável por problemas relacionados a deposição de sólidos. No presente estudo, a tensão interfacial de sistemas contendo asfaltenos e álcoois intermediários foi investigada, usando um tensiômetro óptico. Asfaltenos foram extraídos de um óleo leve da reserva petrolífera nacional e dissolvidos em tolueno. Os resultados mostram uma continua redução da tensão interfacial com o tempo, que permite inferir a cinética de adsorção de moléculas à interface. A presença de álcoois acentua a redução da tensão interfacial da solução, até uma concentração limite, permitindo inferir a presença de álcoois na interface.
\end{abstract}

\section{INTRODUÇÃO}

Surfactantes são moléculas com habilidade de reduzir a tensão interfacial. Por sua característica anfifílica, sua parte hidrofílica tem uma forte afinidade pela água e sua parte hidrofóbica tem afinidade com o óleo, adsorvendo sobre a interface quando em solução (Santos, 2007; Shaw, 1977; Mosayeb e Abedini, 2012). Um mecanismo de associação de surfactantes em meio aquoso é a formação de micelas, agregados organizados, em que as cadeias de hidrocarbonetos liofóbicas se orientam para o interior da micela, enquanto que os grupos hidrofílicos ficam em contato com o solvente externo polar. A concentração em que se inicia a formação de micelas se chama Concentração Micelar Crítica (CMC) (Shaw, 1977; Mosayeb e Abedini, 2012).

Asfaltenos são surfactantes naturais do petróleo, mas estes não apresentam uma concentração micelar crítica (Loh et al., 2007). Eles são definidos como uma fração do petróleo insolúvel em nalcanos de baixo ponto de ebulição (ex: n-pentanos e n-heptanos) e são solúveis em tolueno, benzeno, dissulfeto de carbono, clorofórmio e outros hidrocarbonetos clorados (Santos, 2007; Poteau et al., 2005; Quintero, 2009). A fração asfaltênica é composta por diferentes espécies moleculares, sendo estas caracterizadas pela presença de anéis aromáticos policondensados ligados a cadeias alifáticas laterais (Loh et al., 2007). Métodos analíticos revelam que asfaltenos são compostos basicamente por carbono e hidrogênio e, em menor proporção, por heteroátomos tais como nitrogênio e enxofre, contendo ainda grupos ácidos e básicos (Santos, 2007). Embora dependa do processo de extração e das características do petróleo, a concentração de asfaltenos no petróleo são normalmente abaixo de $10 \%$ em massa (Loh et al., 2007). 
Asfaltenos tem sido relacionado com a deposição de sólidos em vários processos de extração e produção do petróleo. Em alguns casos, depositam-se nas rochas dos reservatórios limitando a recuperação de frações de petróleo, em outros casos entopem as tubulações, fazendo com que seja necessário periódicas limpezas no sistema (Loh et al., 2007). Novas tecnologias para o transporte de petróleo estão sendo desenvolvidas, sendo uma delas a formulação de emulsões óleo em água (Santos, 2007), mas estas também são afetadas pela presença de asfaltenos e outros componentes polares do petróleo. Estudos recentes mostram que emulsões contendo surfactantes e álcoois intermediários apresentam um resultado ainda mais satisfatório, reduzindo a tensão interfacial e aumentando a estabilidade da emulsão (Santos et al., 2011; Santos et al., 2013; Santos, 2007).

Em sistemas óleo e água, a tensão interfacial é causada pela resultante das forças de atração das moléculas. As moléculas da interface são atraídas apenas para direção do interior do líquido e laterais, pois a diferença de polaridade dos líquidos impede que haja atração idêntica das moléculas da água pelas do óleo e, vice-versa. Enquanto que as moléculas no interior do líquido são atraídas em todas as direções pela sua vizinhança tendo a resultante das forças igual à zero. Como consequência, a força resultante é direcionada para o interior do líquido, produzindo a tensão interfacial (Shaw, 1977).

Tendo em vista estas observações, o estudo busca avaliar a variação de tensão superficial e interfacial de soluções orgânicas de asfaltenos em presença de álcoois e estudar se o álcool atua como cossurfactante, auxiliando o surfactante na redução da tensão interfacial, diminuindo os efeitos repulsivos entre as moléculas, facilitando a adsorção do surfactante.

\section{METODOLOGIA EXPERIMENTAL}

\subsection{Materiais}

(i) Óleo cru oriundo da reserva petrolífera nacional, sendo a gravidade API ${ }^{1}$ de 26,8 , viscosidade dinâmica de 47,740 mPa.s e densidade de $0,8906 \mathrm{~g} / \mathrm{cm} 3$, a $20^{\circ} \mathrm{C}$;

(ii) Solventes orgânicos (Tolueno, n-pentano, n-butanol)

$$
{ }_{1}^{o} A P I=\frac{141,5}{d_{60 / 60}}-131,5
$$




\subsection{Métodos}

Extração de asfaltenos do petróleo: A extração de asfaltenos do petróleo emprega o procedimento IP 143, o qual se baseia na separação por solubilidade. Pesa-se $10 \mathrm{~g}$ de petróleo e mistura-se com 400mL de floculante (n-pentano) na proporção 40:1 (volume de floculante por grama de óleo). A mistura é mantida sob agitação por 8 horas em uma placa de agitação a temperatura ambiente. O sólido precipitado é filtrado a vácuo. Em seguida, o sólido retido no papel de filtro é purificado por extração Soxhlet, onde o sólido é lavado com o floculante. Deixa-se refluxando até que o extrato esteja totalmente límpido e translúcido, garantindo assim menor risco de presença de impurezas. A massa do sólido é então determinada e quantificada.

Determinação da tensão superficial/interfacial de soluções de asfantenos:

(i) Tensão superficial de soluções de asfaltenos: determinação da concentração de agregação.

(ii) Medidas de tensão superficial de soluções de asfaltenos com álcoois: $\mathrm{Na}$ concentração de $15 \mathrm{~g} / \mathrm{L}$ de asfaltenos, determina-se a tensão superficial variando a concentração de butanol em 2, 4, 6 e $8 \%$ em relação à massa de tolueno.

Para medir a tensão superficial utiliza-se um tensiômetro ótico Theta Lite (KSV, Finlândia) que captura imagens sucessivas da gota do fluido em análise. Sendo que, uma gota é formada na ponta de uma seringa preenchida pela fase óleo (solução de asfaltenos em tolueno). A forma da gota é analizada pelo sistema utilizando o método ADSA (axyssimetric drop shape analysis), baseado na equação de Laplace para determinar a tensão interfacial como função do tempo.

\section{RESULTADOS E DISCUSSÃO}

\subsection{Caracterização da amostra}

Para caracterizar o óleo, utilizou-se um Viscosímetro (SMV 3000), com o qual se pode determinar as viscosidades dinâmicas e cinemáticas, além da densidade para várias temperaturas.

Com os dados da tabela coletados, construiu-se o gráfico exposto na Figura 1. No gráfico são apresentadas a variação da viscosidade dinâmica e da densidade em função da temperatura. Os dados mostram que a densidade decresce linearmente com o aumento da temperatura enquanto a viscosidade apresenta um decréscimo representado por uma curva exponencial. As respectivas equações são apresentadas nas equações 1 e 2 , sendo que em ambos os casos o coeficiente de ajuste $\left(\mathrm{R}^{2}\right)$ foi maior que 0,97 .

Densidade em função da temperatura

$\rho=0,905-6,712 \cdot 10^{-4} T$ 
Viscosidade dinâmica em função da temperatura

$$
\mu=210,17 \mathrm{e}^{-0,059 \mathrm{~T}}
$$

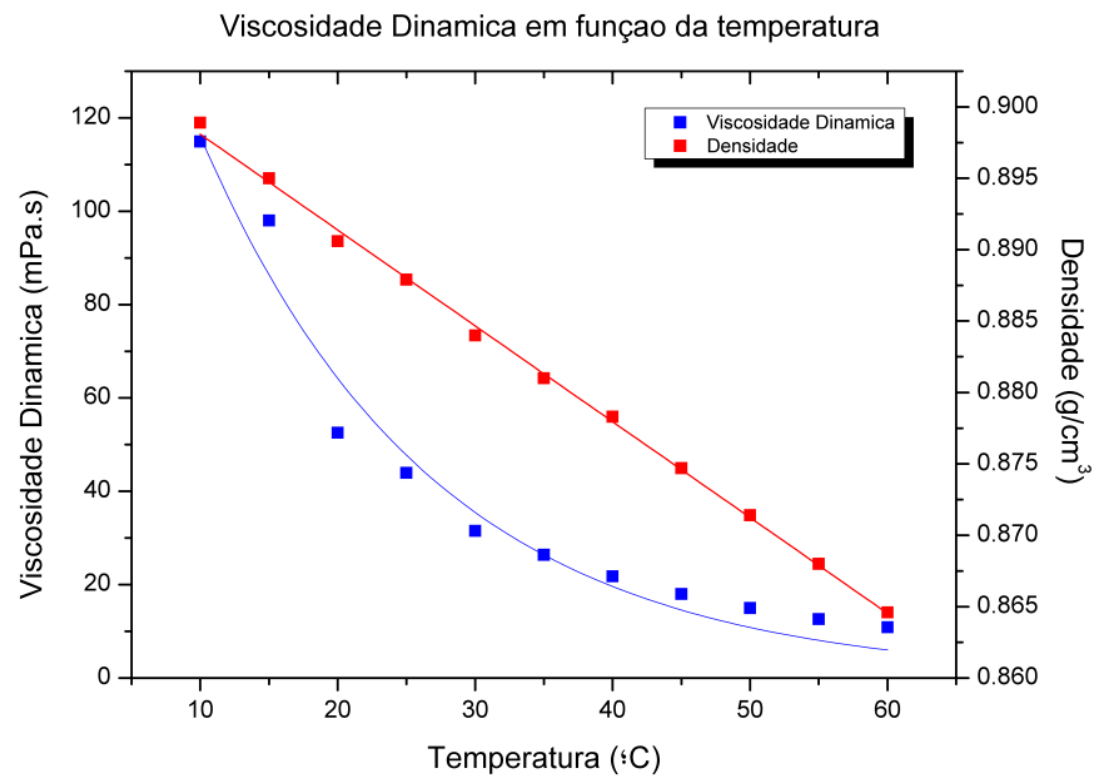

Figura 1. Viscosidade dinâmica e Densidade em função da temperatura

O coeficiente angular da equação referente à densidade em função da temperatura $\left(-6,712.10^{-}\right.$ $\left.{ }^{4}\right)$ revela que a densidade varia minimamente conforme a mudança de temperatura, enquanto que a viscosidade dinâmica diminui de forma mais acentuada com o acréscimo de temperatura. Foi observada uma redução de cerca de 10 vezes na viscosidade para um aumento de $50{ }^{\circ} \mathrm{C}$. A redução da viscosidade dinâmica com o aumento da temperatura se deve sobretudo ao aumento das distâncias intermoleculares que reduz a força de atração entre as moléculas. $\mathrm{O}$ efeito da temperatura sobre a viscosidade pode ser modelado pela equação de Arrhenius, dada pela equação 3 .

$$
\mu=\mu_{0} e^{\frac{E_{a}}{R T}}
$$

$\mathrm{E}_{\mathrm{a}}$ corresponde à energia de ativação ao fluxo; $\mathrm{R}$, à constante universal dos gases ideais; $\mathrm{T}$, à temperatura absoluta; $\mu$, à viscosidade dinâmica e $\mu_{0}$ à viscosidade dinâmica quando a temperatura tende a infinito. A energia de ativação ao fluxo é uma medida da sensibilidade da viscosidade diante da variação de temperatura, de forma que quanto maior a energia de ativação, maior a influência da temperatura.

Linearizando a equação 3 temos um relação entre $\ln \mu$ e $1 / \mathrm{T}$, equação 4 , o que permitiu a construção do gráfico exposto na figura 2. 
$\ln \mu=-18,06+\frac{4473,67}{T}$

In da viscosidade em funçao do inverso da temperatura

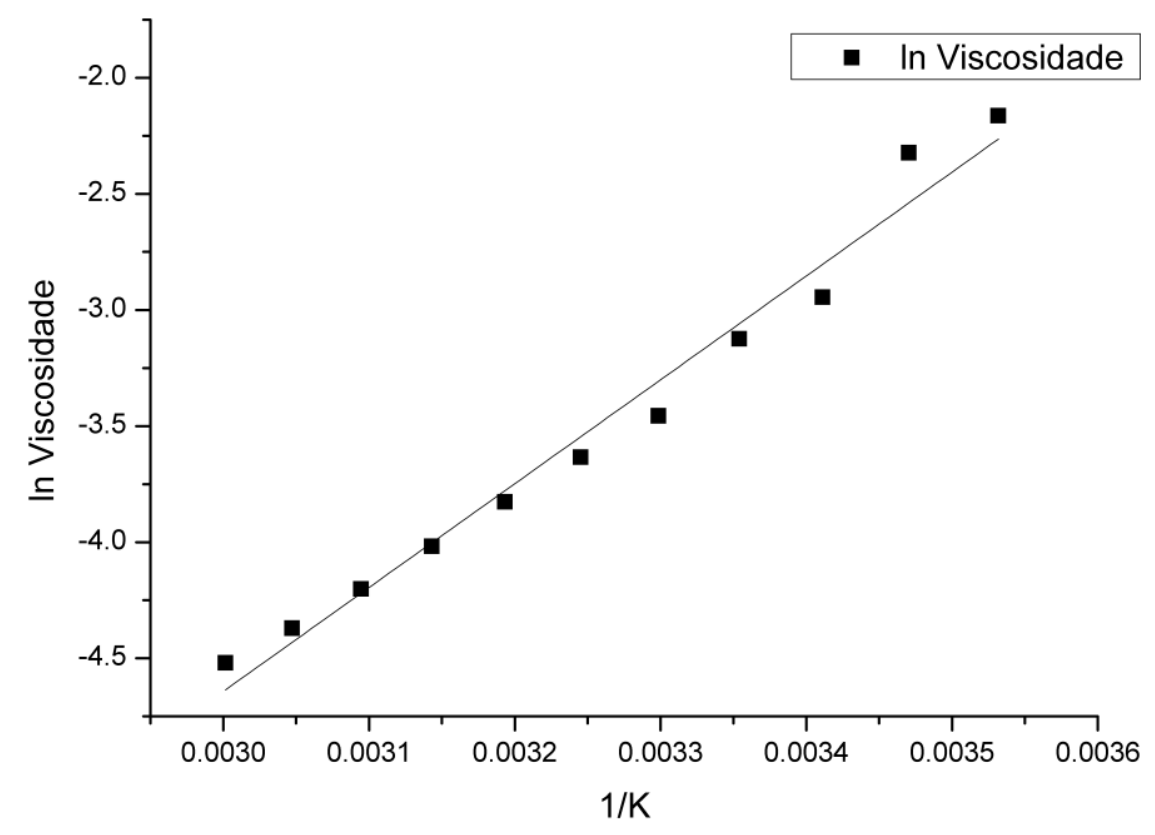

Figura 2. Linearização da curva de viscosidade dinâmica.

$O$ coeficiente angular é numericamente igual à relação $\left(E_{a} / R\right)$ de forma que a Energia de ativação de fluxo do petróleo utilizado é de $37,2 \mathrm{~kJ} / \mathrm{mol}$.

\subsection{Determinação da concentração de agregação}

Por meio do procedimento descrito na seção 2.2, foram extraídos $0,541 \mathrm{~g}$ de asfaltenos. Cada extração resultou em uma massa média de $0,135 \mathrm{~g}$ de asfaltenos. A porcentagem em massa média de asfaltenos foi de 1,3\% em relação à massa de óleo. Estes valores refletem uma baixa porcentagem de asfaltenos, típica de óleos leves, se comparada àquelas de óleos pesados, tal como reportado por Loh et. al. (2007).

Foram preparadas soluções de asfaltenos C5I em tolueno nas concentrações de 5 a $30 \mathrm{~g} / \mathrm{L}$. A tensão superficial dinâmica para cada uma delas foi então determinada. A curva tensão superficial em função do tempo se assemelha a uma exponencial, sendo que em um determinado momento se estabiliza o que confere o valor estabelecido para cada concentração, conforme mostra cada ponto experimental da figura 3 . 


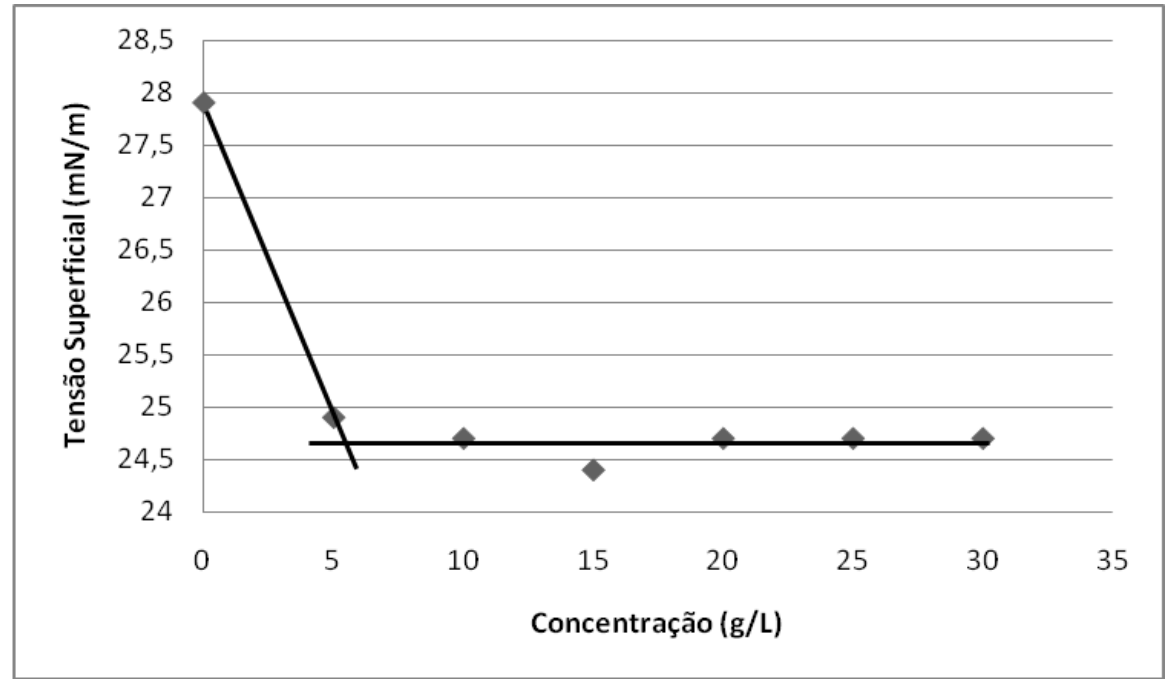

Figura 3. Tensão Superficial em função da concentração de Asfaltenos.

Através da tensão superficial em função da concentração pode-se determinar a concentração de agregação, sendo ela a concentração em que se inicia a formação de agregados organizados. A concentração de agregação, extraída da curva tensão superficial versus o logaritmo da concentração da solução, foi de aproximadamente 5,5 g/L. Loh et. al. (2007) reporta valores de concentração de agragação superiores a $10 \mathrm{~g} / \mathrm{L}$ para asfaltenos insolúveis em pentano. Porém como este estudo trata de asfaltenos provenientes de um óleo leve, é razoável que o comportamento dinâmico de asfaltenos em solução seja inferior àquele mostrado em óleo pesados (Loh et al., 2007).

\subsection{Efeito de adição de alcoóis sobre a tensão superficial}

Para avaliar o efeito da adição de alcoóis sobre a tensão superficial utilizou-se uma solução de asfaltenos C5I em tolueno $15 \mathrm{~g} / \mathrm{L}$, variando a concentração de n-butanol de 0 a $8 \%$ em massa em relação à massa de tolueno na solução. Assim como na determinação da concentração de agregação, realizou-se a medida da tensão superficial para cada concentração conforme mostra a figura 4 .

A Figura 4 mostra que a tensão superficial tende a diminuir à medida que a concentração de álcool aumenta, até a concentração de $4 \%$ em massa, sendo que em 6 e $8 \%$ em massa a tensão superficial torna a ser de $24,4 \mathrm{mN} / \mathrm{m}$, similar aquela encontrada na ausência de n-butanol. Este fato pode ser explicado pela fraca interação entre o butanol e óleo, devido ao pequeno tamanho de cadeia do álcool que impede a redução efetiva da tensão superficial. O efeito do álcool está relacionado com sua solubilidade na fase óleo e com sua interação com a superfície (Santos, 2007). 


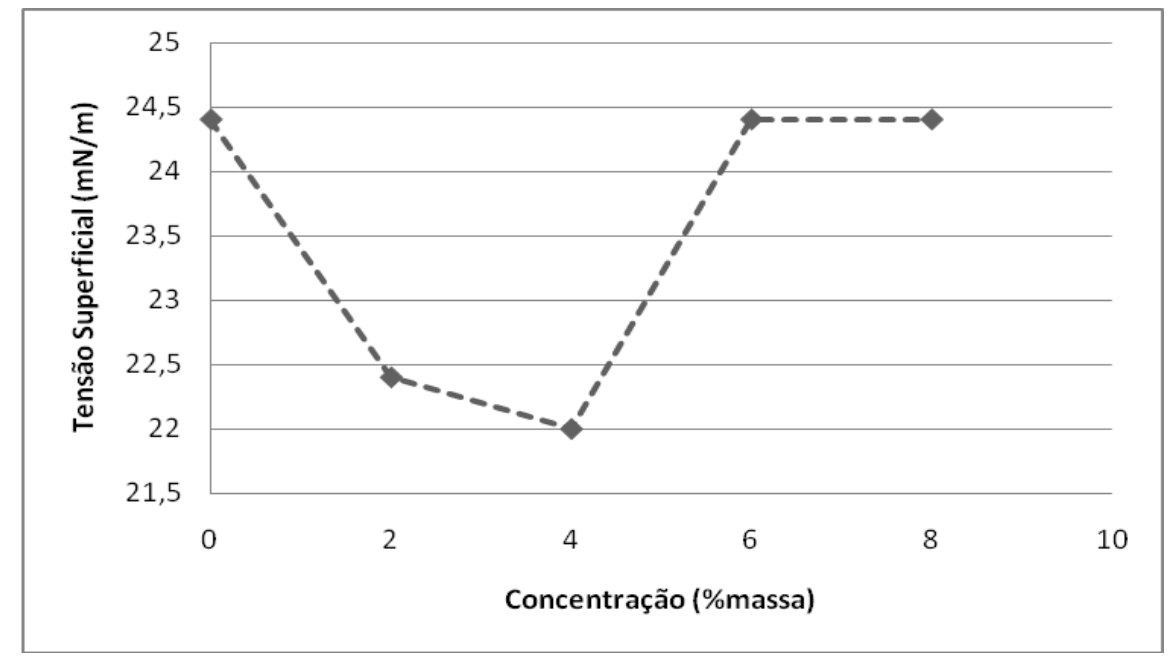

Figura 4. Tensão Superficial em função da concentração de n-butanol.

\section{CONCLUSÃO}

O óleo leve estudado neste projeto possui gravidade API de 26,8, viscosidade dinâmica de $47,740 \mathrm{mPa}$.s e densidade de $0,8906 \mathrm{~g} / \mathrm{cm} 3$, a $20^{\circ} \mathrm{C}$. Sendo que a viscosidade varia exponencialmente com a temperatura, enquanto que a densidade sofre uma variação linear. Através da equação de Arrhenius, pode-se determinar a energia de ativação de fluxo deste óleo como $37,2 \mathrm{~kJ} / \mathrm{mol}$. Obteve-se $1,30 \%$ em massa de asfaltenos C5I. A tensão superficial de soluções de asfaltenos é reduzida de 28 $\mathrm{mN} / \mathrm{m}$ para $24 \mathrm{mN} / \mathrm{m}$, conforme a concentração de asfaltenos varia de 0 a $30 \mathrm{~g} / \mathrm{L}$. A concentração de agregação é de aproximadamente 5,5 g/L. Com a adição de n-butanol de 0 a $8 \%$ em massa mantendo a solução de $15 \mathrm{~g} / \mathrm{L}$ de asfaltenos, a tensão superficial varia de 22 a 24,5 mN/m, uma vez que a tensão superficial diminui até a concentração de $4 \%$ em massa de butanol e torna ao valor inicial nas concentrações de 6 e $8 \%$ em massa, efeito explicado pela curta cadeia alquil do butanol que reduz sua interação com o óleo. 


\section{REFERÊNCIAS}

LOH, W.; SANTOS, R.G., MOHAMED, R.S. Crude oil asphaltenes: Colloidal aspects. In: P. Somasundaram. (Org.). Encyclopedia of Colloid and Surface Science. Nova York: Taylor \& Francis, $1: 1,1-18,2007$.

MOSAYEB, A.; ABEDINI, R. The effect of non-ionic surfactants on the interfacial tension between crude oil and water. Petroleum \& Coal, 54, 110-113, 2012.

POTEAU, S.; ARGILlIER, J.; LANGEVIN, D.; PINCET, F.; PEREZ, E. Influence of pH on Stability and Dynamic Properties of Asphaltenes and Other Amphiphilic Molecules at the Oil-Water Interface. Energy \& Fuels, 19, 1337-1341, 2005.

QUINTERO, L. Fracionamento e análise de asfaltenos extraídos de petróleos brasileiros. Tese (Doutorado em ciências) - Universidade Federal do Rio de Janeiro, 15-35, 2009.

SANTOS, R.G. Formulação e propriedades reológicas e coloidais de emulsões óleo em água para a aplicação em processos de escoamento de óleos pesados, 31- 33, 2007.

SANTOS, R.G., BRICEÑO, M. I., BANNWART, A.C., LOH, W. Physico-chemical properties of heavy crude oil-in-water emulsions stabilized by mixtures of ionic and non-ionic ethoxylated nonylphenol surfactants and medium chain alcohols. Chemical Engineering Research and Design 89, 957-967, 2011.

SANTOS, R.G., BANNWART, A. C., LOH, W. Phase segregation, shear thinning and rheological behavior of crude oil-in-water emulsions. Chemical Engineering Research and Design, 1-8, 2013.

SHAW, D.J. Introducción a la química de superficies y coloides. Editora Alhambra. Madrid, España, $65-113,1977$. 